\title{
Author Correction: Genomic epidemiology reveals multiple introductions of SARS-CoV-2 from mainland Europe into Scotland
}

Ana da Silva Filipe (1), James G. Shepherd (1D), Thomas Williams (1), Joseph Hughes (1), Elihu Aranday-Cortes (1),

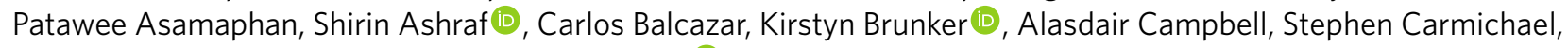

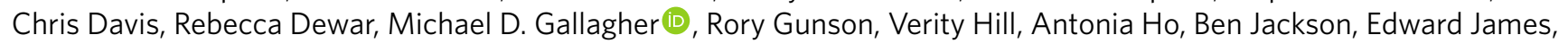
Natasha Jesudason, Natasha Johnson (1), E. Carol McWilliam Leitch D, Kathy Li, Alasdair MacLean, Daniel Mair, David A. McAllister, John T. McCrone, Sarah E. McDonald, Martin P. McHugh (1), A. Keith Morris, Jenna Nichols,

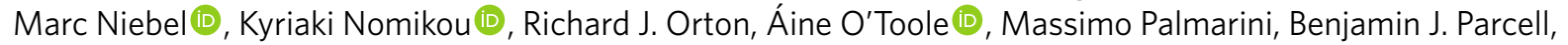
Yasmin A. Parr (D), Andrew Rambaut (1), Stefan Rooke, Sharif Shaaban (D), Rajiv Shah (D), Joshua B. Singer, Katherine Smollett, Igor Starinskij®i), Lily Tong, Vattipally B. Sreenu (D), Elizabeth Wastnedge (D), The COVID-19 Genomics UK (COG-UK) Consortium, Matthew T. G. Holden (1), David L. Robertson (1), Kate Templeton and Emma C. Thomson (1)

Correction to: Nature Microbiology https://doi.org/10.1038/s41564-020-00838-z, published online 21 December 2020.

In the Supplementary Information PDF originally published with this Article, the surname of the consortium member Dimitris Grammatopoulos was misspelt 'Gramatopoulos'. This error has now been corrected.

${ }^{*} \mathrm{~A}$ list of authors and their affiliations appears online.

Published online: 27 January 2021

https://doi.org/10.1038/s41564-021-00869-0

(c) The Author(s), under exclusive licence to Springer Nature Limited 2021 